\title{
Vergleichende und kontrastive Linguistik Deutsch-Spanisch: eine Bestandsaufnahme
}

\author{
Jossé Antonio Calañas ConTinewTe · Hang FerRrer Mora \\ Universitat de València
}

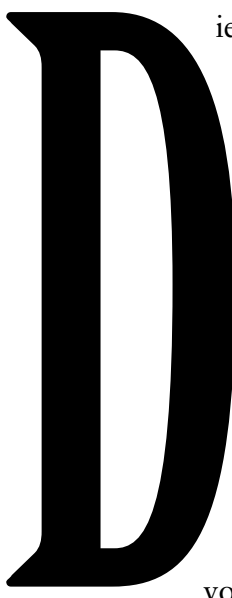

ie menschliche Sprache ist ein vielseitiges Phänomen ${ }^{1}$. Das kann als unbestrittene Annahme gelten, denn man denkt in einer Sprache, d.h., erst durch Sprache kann der Mensch die Welt erfahren und erkennen, durch Sprache wird diese Welt codiert und weitergegeben. Wenn man sich im positivistischen Sinne wissenschaftlich mit einer Sprache beschäftigen will, ergeben sich unzählige Möglichkeiten der Betrachtung. So sieht sich der angehende wie der erfahrene Sprachwissenschaftler mit etlichen Grundsatzfragen konfrontiert, ehe er sich dieser Arbeit annehmen kann. Diese Grundsatzfragen dienen vor allem der Rahmensetzung, der Kontextualisierung des Forschungsvorhabens. Man stellt sich Fragen nach dem Forschungsgegenstand (was), nach der Methodologie (wie) und gegebenenfalls auch nach dem Zweck (wozu) der Forschung: Der Rahmen bildet keine Einschränkung, er stellt eher die unentbehrlichen Richtlinien dar, die auf eine fruchtbare Arbeit hinzielen.

In diesem Beitrag wird über einen der vielen möglichen Ansätze in der linguistischen Forschung berichtet, nämlich über den komparatistischen. Aus diesem Ansatz ergeben sich zwei -wenn auch doch sehr nahe stehende- Hauptlinien, die im Folgenden dargestellt werden: die vergleichende auf der einen Seite, die kontrastive Sprachwissenschaft auf der anderen: Beide Richtungen verfolgen andere Ziele mit unterschiedlichen Methodologien, beide können wissenschaftlich gerechtfertigt werden. Im Anschluss an die Darstellung von kontrastiver und vergleichender Linguistik wird die Arbeit in diesen Teildisziplinen veranschaulicht.

\section{Womit beschäftigen sich die Kontrastive und die Vergleichende Linguistik?}

Jede vergleichende Arbeit erfordert die Beschäftigung mit mindestens zwei Gegenständen, sonst wäre kein Vergleich möglich. Im Falle der kontrastiven oder der vergleichenden Linguistik besteht das Forschungsobjekt aus mindestens zwei Sprachen; je mehr Sprachen, umso komplexer wird die Forschung auf dem Gebiet dieser Disziplin. Der linguistische Vergleich steht heute auf der Grundlage einer langen Tradition, älter als andere Teildisziplinen wie z.B. die Pragmatik, er kann aber immer noch als junge Disziplin angesehen werden, denn an Aktualität ist nichts eingebüßt worden.

Kontrastive Linguistik und vergleichende Linguistik sind zwei Bezeichnungen, die leicht irreführend wirken, denn beide sind oft dazu verwendet worden, scheinbar nahe stehende Arbeiten zu benennen. Es kann jedoch ein erster Unterschied festgelegt werden: die vergleichende Linguistik setzt sich als Hauptziel die Suche nach Universalien und wird deshalb mit Studien zur Sprachtypologie in Verbindung gebracht, so Moreno Cabrera (1997:13):

«La lingüística tipológica y universalista es aquella rama de la ciencia del lenguaje que se ocupa de estudiar las semejanzas y las diferencias entre las lenguas humanas. El objeto de estudio de esta disciplina es la totalidad de las lenguas humanas y no una lengua en particular.»

Universalien hängen dann mit Typologie oder Sprachtypologie eng zusammen. Eine Definition von Sprachtypologie bieten Haspelmath et al. (2001:VII): «Die Sprachtypologie beschäftigt sich mit den Gemeinsamkeiten und Unterschieden zwischen den Sprachen der Welt, insbesondere mit den Regelmäßigkeiten und Grenzen, die innerhalb der Verschiedenheit beobachtet werden können.»

Bei der Typologie handelt es sich um keine neue Disziplin innerhalb der Linguistik. Im 19. Jahrhundert existierte sie schon und war eher morphologisch orientiert: Sprachen wurden nach morphologischen Kriterien (Vorhandensein von Flexion, Wortbildung usw.) in starre Sprachtypen klassifiziert. Im 20. Jh. beschäftigt sich die strukturelle Sprachtypologie dagegen mit Einzelmerkmalen auf allen sprachlichen Ebenen. Nach wie vor trägt die Sprachtypologie jedoch durch den Vergleich verwandter Sprachen und Sprachfamilien zur Erforschung sprachlicher Universalien bei.

Andererseits beschäftigt sich die kontrastive Linguistik mit dem Vergleich eines oder mehrerer bestimmter Aspekte in zwei Sprachen bzw. innerhalb einer reduzierten Sprachgruppe (vgl. Pörings/Schmitz 1999:258).

Nachdem Gegenstand und Ziele beider Teildisziplinen einigermaßen dargelegt wurden, wenden wir uns einigen der zentralen Fragen zu, die sie beschäftigen:

1. Wie viele Sprachen gibt es auf der Welt und wie kann man deren Zahl errechnen?

2. Wie können die Varietäten einer Sprache bestimmt werden? Damit verbunden auch, wie kann man diesen Varietäten dem Status Sprache oder Dialekt zuordnen?

3. Welche sind die wichtigsten Sprache der Welt?

4. Welche Kriterien sollen herangezogen werden, um Sprachvergleiche zu ziehen?

5. Welche Bestandteile einer Sprache kommen für einen Vergleich in Frage? 


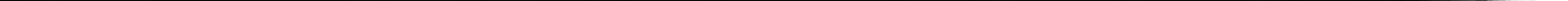


Sprachen addieren... aber auch substrahieren

Trotz immer währenden Fortschritts erweist es sich immer noch als schwierig, eine ungefähre Anzahl der Sprachen anzugeben, die heutzutage in der Welt gesprochen werden. Das Metzler Lexikon Sprache (Glück (Hrsg.), 2000: 650) gibt an, die Zahl der bekannten natürlichen Sprachen belaufe sich auf über 5000, doch soll diese nur als grobe Richtzahl gelten, denn es wird davon ausgegangen, dass in schwer zugänglichen Gegenden, wie etwa dem Regenwald am Amazonas oder auf Papua-Neuguinea, noch unklassifizierte Sprachen existieren.

Grimes (1992) spricht in seinem Ethnologue von 6528 klassifizierten Sprachen, deren geografische Verteilung auf den Kontinenten in Tabelle I (vgl. Moreno Cabrera 1997:14) dargestellt wird.

\begin{tabular}{lcc} 
Kontinent & Anzahl Sprachen & $\%$ \\
\hline Afrika & 1995 & $30 \%$ \\
Amerika & 949 & $15 \%$ \\
Asien & 2034 & $31 \%$ \\
Europa & 209 & $3 \%$ \\
Ozeanien & 1941 & $21 \%$ \\
\hline
\end{tabular}

Tabelle I: Distribution von Sprachen auf Kontinente

Die Feldforschung besteht im Feststellen, Registrieren und Analysieren einer natürlichen Sprache entweder direkt in ihrem Verbreitungsgebiet oder durch den Zugriff auf Muttersprachler. Wenn es aber darum geht, eine unbekannte Sprache ins Visier des Forschers zu nehmen, weist diese Methode folgende Schwierigkeiten auf:

Dem Forscher mangelt es an den erforderlichen kognitiven Mitteln, die betroffene Sprache von anderen Varianten bzw. von ähnlichen Sprachen auseinanderzuhalten;

Oft verfügt der Forscher über keine Mittel, die Sprache zu beschreiben, nicht einmal um diese zu erlernen (was übrigens auch erfahrenen Linguisten schwer fällt). Aus diesem Grund werden etliche Phänomene unbeachtet bleiben;

Sollte der Sprachforscher die betroffene Sprache erlernen wollen, so wird dieses Vorhaben sehr zeitaufwendig, wenn eine hohe Sprachkompetenz angestrebt wird. Diese Zeitspanne vergrößert sich mit zunehmendem typologischem Abstand zwischen der Muttersprache des Forschers und der zu erlernenden/beobachtenden Sprache.

Natürliche Sprachen ähneln lebendigen Organismen: sie kommen zur Welt, entwickeln sich und sterben aus. Beispiele für ausgestorbene Sprachen sind: das Dalmatinische oder das Manx (Sprache der Einwohner der Insel Man); andere ausgestorbene Sprachen, die heutzutage immer noch -wenn auch in reduzierter Form- gebraucht werden sind das Altgriechische oder Latein.

Ein weiteres Problem stellt die Abgrenzung einer Sprache als solche dar, d. h., diese vor anderen verwandten Sprache zu unterscheiden bzw. sie als dialektale Variante zu betrachten, ein nicht zu unterschätzendes Unternehmen, wie im Folgenden gezeigt wird.

\section{Sprache oder Dialekt?}

Diese Frage, wie so viele andere in der Linguistik, kann entwe- der aus der synchronen oder der diachronen Perspektive beantwortet werden, und die gewählte Perspektive wird selbstverständlich die Antwort bedingen.

Synchronisch gesehen ist die Frage Sprache oder Dialekt immer noch aktuell, man nehme sich nur als Beispiel die Situation in Spanien! Doch oft sprengt das Problem der Sprachklassifizierung den Rahmen der Philologie. Auf der Iberischen Halbinsel findet man typologisch isolierte Sprachen wie das Baskische neben anderen, die sich typologisch nahe stehen (Unterfamilie iberoromanisch: Spanisch, Portugiesisch, Katalanisch); in anderen Fällen wird von anderen um den Status ,Sprache' gekämpft: das wäre der Fall vom Valencianischen in Bezug auf das Katalanische oder vom Galizischen in Bezug auf das Portugiesische. Valencianisch oder Mallorquinisch werden philologisch als Dialekte des Katalanischen betrachtet, was aus sprachsoziologischen wie geografischen Kriterien durchaus vertretbar ist. Die philologischen Kriterien weichen jedoch hier von den Gefühlen der Völker und von den Positionen einiger Politiker ab: Das hat zur Gründung der Acadèmia Valenciana de la Llengua (Valencianische Sprachakademie) geführt, was noch mehr zur allgemeinen Verwirrung in diesem Bereich beiträgt. Natürliche Sprachen kennen keine Grenzen... bis man sie in Schranken verweist.

Verfolgt man dieses Thema und bezieht es auf Lateinamerika, so stellt man fest: Niemand stellt in Frage, dass in Brasilien Portugiesisch und sonst fast überall auf diesem Kontinent Spanisch gesprochen wird, unabhängig von der Tatsache, dass die dort auf-

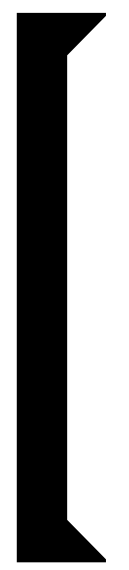

Diachronisch gesehen erschwert die natüriliche Evolution von Sprachen die Antwort auf die Frage nach der Ceburrstunde einer Einzelsprache, wann diese sich von der «Irsprache” endoültig trennt.

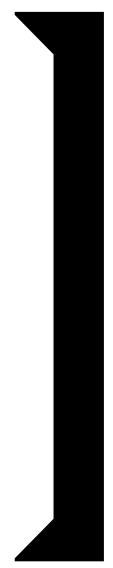

kommenden sehr reichhaltigen Varietäten abweichende Entwicklungen erfahren. Wenn sich diese Entwicklung von der ursprünglichen Sprache weg fortsetzt, wird man irgendwann zu einem Punkt gelangen, ab dem von selbständigen Sprachen die Rede sein wird, wie im Fall der romanischen Sprachen hinsichtlich des Lateins.

Diachronisch gesehen erschwert die natürliche Evolution von Sprachen die Antwort auf die Frage nach der Geburtstunde einer Einzelsprache, wann diese sich von der «Ursprache» endgültig trennt, denn die Evolution ist ein Kontinuum, während präzise Augenblicke auf dieser kontinuierlichen Linie nur durch synchrone Querschnitte erfassbar sind.

Sprachen auf der Waage: Welche sind die wichtigsten Sprachen der Welt? Um auf diese Frage eingehen zu können, muss man als Erstes die 
Anzahl der Sprecher in Betracht ziehen, sowohl die der Muttersprachler als auch die derjenigen, die diese Sprache als Zweitoder Fremdsprache sprechen. Wenn man sich für das Kriterium der Anzahl von Muttersprachlern entscheidet, sieht die Gewichtung in etwa so aus (nach Angaben von Grimes 1996):

\begin{tabular}{lc} 
Sprache & Sprecher (Mio.) \\
\hline Mandarin & 885 \\
\hline Englisch & 450 \\
\hline Spanisch & 266 \\
Hindi/Urdu & 233 \\
Malaiisch Indonesisch & 193 \\
Bengalesisch & 181 \\
\hline Portugiesisch & 175 \\
\hline Russisch & 160 \\
\hline Arabisch & 139 \\
\hline Japanisch & 126 \\
\hline Französisch & 122 \\
\hline Deutsch & 118 \\
\hline
\end{tabular}

Tabelle II: Meistgesprochene Muttersprachen

Diese «Hitparade» würde jedoch anders aussehen, wenn man zu anderen Kriterien greift: Anzahl der Länder, wo eine Sprache Amtssprache ist, Kontinente, in denen sie gesprochen wird usw. (vgl. Pörings/Schmitz 1999: 248):

\begin{tabular}{lccc} 
Sprache & Amtsprache in Ländern & Erste Sprache & Kontinente \\
\hline Englisch & 47 & 300 & 5 \\
\hline Französisch & 30 & 68 & 3 \\
\hline Arabisch & 21 & 139 & 2 \\
Spanisch & 20 & 266 & 2 \\
\hline Portugiesisch & 7 & 175 & 3 \\
Deutsch & 5 & 118 & 1 \\
\hline Malaiisch Indonesisch & 4 & 193 & 1 \\
\hline
\end{tabular}

Tabelle III: Meistgesprochene Sprachen

Unbestrittene Tatsache ist heutzutage die Rolle des Englischen als lingua franca, eine Rolle, die früher das Lateinische oder das Französische gespielt haben. Es fehlt jedoch an zuverlässigen statistischen Erhebungen über die Anzahl der Lerner von Englisch als Fremd- oder Zweitsprache. Dass es sich hier um variable Zahlen handelt, lässt sich an Ereignissen wie in Osteuropa erkennen, wo ab 1991, nach dem Zusammenbruch des sowjetischen Systems Russisch als erste Fremdsprache durch Englisch verdrängt wurde.

\section{Kriterien zum Vergleich und Kontrast von Sprachen}

Wenn es darum geht, Sprachvergleiche zu ziehen, kann man auf Anhieb zwei große Gruppen von Faktoren unterscheiden:

- Innersprachliche Faktoren, die sprachimmanent sind: formale, phonologische, semantische Merkmale usw.

- Außersprachliche Faktoren: soziale, geografische usw. Innersprachlich gesehen führt der Vergleich zwischen Sprachen zur Beobachtung von den Ähnlichkeiten, die sich auf dem Be- reich der klassischen linguistischen Disziplinen wie Phonologie, Lexikologie, Morphologie und Syntax vollziehen. Hier geht es eigentlich um formale, strukturelle und semantische Fragen. Oft werden Sprachen, die auf verschiedenen Feldern Ähnlichkeiten aufweisen, Familien zugeordnet, die von einer gemeinsamen UrSprache abgeleitet sind. Dabei kann man sich nicht nur auf die synchronische Betrachtung beschränken, man muss vielmehr den Stand der Sprachentwicklung diachronisch festlegen, oft werden ganze Sprachen wie das Indogermanische rekonstruiert.

Diese Art der Sprachenklassifizierung, die Greenberg (1996) genetische Klassifikation nennt, basiert auf der Entwicklung dieser Sprachen. Familien können eingeteilt werden in Subgruppen bzw. Subfamilien (z.B. die galo-romanische Gruppe mit Französisch und Provenzalisch), aber auch in größere Einheiten, in eine Makrofamilie (z.B. die Familie der baltischen Sprachen, die auch Lettisch und Litauisch einschließt, bildet zusammen mit der slawischen Familie die balto-slawische Makrofamilie; vgl. Moreno Cabrera 1997: 42). Makrofamilien lassen sich wiederum in Phyla gruppieren, wie das Indoeuropäische; Mehrere Phyla bilden ein Makrophylum und diese können zu einem Megaphylum zusammengetan werden, wenn man sie für genetisch verwandt hält. Bomhard und Kern (1994) behaupten, es existiert das euroasiatische Makrophylum, dem das indoeuropäische, das uralische sowie das altaische Phylum angehören; dieselben Autoren sprechen von dem nostratischen Megaphylum, zu dem das euroasiatische Makrophylum, die damit verwandte kartuelische Familie (Georgianisch) sowie das afroasiatische Phylum -das die semitische Familie einschließt-gehören. Als Beispiel zitieren wir die genetische Klassifikation des Spanischen nach Moreno Cabrera (1997: 43):

\section{Megaphylum: Nostratisch \\ Makrophylum: Euroasiatisch \\ Phylum: Indoeuropäisch \\ Makrofamilie: Italisch \\ Familie: Romanisch \\ Subfamilie: Iberoromanisch}

Die typologische Klassifizierung wird aufgrund grammatischer Merkmale, die einer Gruppe von Sprachen gemeinsam sind, durchgeführt. Der Tabelle IV kann eine Zusammenfassung der Merkmale entnommen werden, die es beim linguistischen Vergleich zu beachten gilt (Décsy 1983 apud Moreno Cabrera 1997: 47):

\begin{tabular}{|c|c|}
\hline 1. Indemas & Rasgos que aparecen sólo en una lengua \\
\hline 2. Cardemas & $\begin{array}{l}\text { a. Unemas: Rasgos universales, comunes a todas las lenguas. } \\
\text { b. Genemas: Rasgos comunes a los miembros de una misma } \\
\text { familia de lenguas. } \\
\text { c. Tipemas: Rasgos comunes a diversas lenguas que } \\
\text { pertenecen al mismo tipo lingüístico. } \\
\text { d. Areemas: Rasgos comunes a una serie de lenguas } \\
\text { que comparten la misma área o territorio geográfico. } \\
\text { e. Tijemas: Rasgos en que dos o más lenguas coinciden } \\
\text { de modo casual. }\end{array}$ \\
\hline
\end{tabular}

Tabelle IV: Elemente zum Sprachvergleich 
Wenn man außerlinguistische Faktoren in Betracht zieht, werden Klassifikationen unternommen, denen geografische und territoriale Kriterien zugrunde liegen. Dabei spielen Fragen zu Dialekten, zu staatlichen Grenzen eine wichtige Rolle.

Zwei oder mehr Sprachen teilen sich oft ein Areal, dass entweder durch geografische Begebenheiten, die ein Gebiet umschließen, oder durch auf dem Reißbrett gezeichnete Grenzen festgelegt wird. Ein größeres Gebiet kann eine Familie oder sogar eine Makrofamilie beherbergen. Einige Areale fallen besonders groß aus: Das ist der Fall der Tonsprachen im Südosten Asiens, unter denen sich das Kantonesische und das Mandarin, sowie das Thai und das Vietnamesische befinden (vgl. Bisang 2006).

Sprachen, die geschichtlich in Kontakt gewesen sind, beispielsweise als Folge der Kolonisierung, haben neue Dialekte und Sprachen hervorgebracht: Pidgins und Kreolsprachen haben auch das Interesse der Sprachforscher auf sich gezogen.

Forscher sehen sich auch Problemen ausgesetzt, wenn es darum geht, universalistische Parameter festzulegen. Eins dieser Probleme liegt in der Datenerhebung: man ist auf Informanten angewiesen, und diese machen einen unterschiedlichen Gebrauch von derselben Sprache, was zu unterschiedlichen Auffassungen von Korrektheit führt.

Äpfel und Birnen: Homogenes und Heterogenes vergleichen

Kehren wir zur sprachlichen Perspektive zurück: Welche Aspekte, Phänomene oder Fragen lassen sich vergleichen?

Der Vergleich von zwei Sprachen setzt voraus, dass das zu vergleichende Phänomen über Eigenschaften verfügt, die einen Vergleich mit einer anderen Sprache zulässt. Das mag sogar tautologisch klingen, ist aber gar nicht so einfach, denn eine Sprache kann z.B. ergativisch wie das Baskische sein und die zu vergleichende, sagen wir mal Spanisch, nicht. Will man typologisch arbeiten, so muss man den Vergleich semantisch, kognitiv oder pragmatisch gestalten. Ziel von der Arbeit des Komparatisten ist es, nach dem sprachlichen Ausdruck eines gegebenen Sprachphänomens und nach den Korrelationen unter gewissen innerhalb eines Sprachentyps existierenden Schablonen zu suchen und die Ergebnisse mit der Realisierung in anderen Sprachen in Verbindung zu bringen.

Ausgerechnet hier ist es, wo einige Autoren den Unterschied zwischen kontrastiver und vergleichender Linguistik ansetzen: Die vergleichende Linguistik würde sich mit der typologischen Klassifizierung von Sprachen beschäftigen, während die kontrastive Linguistik sich der Forschung über konkrete Aspekte in zwei oder mehreren Sprachen widmet. Diese theoretische Unterscheidung löst sich jedoch in einer Vielzahl der kontrastiven und vergleichenden Arbeiten auf, die bis jetzt durchgeführt worden sind.

Croft (2003: 14) beschreibt mit folgenden Worten die Strategie, die bei der Forschung auf dem Bereich der Sprachtypologie verfolgt werden soll:

(i) Determine the particular semantic (pragmatic) structure or situation type that one is interested in studying.

(ii) Examine the morphosyntactic construction(s) or strategies used to encode that situation type.

(iii) Search for dependencies between the construction(s) used for that situation and other linguistic factors.
Auf der Suche nach Ähnlichkeiten kann auch oft beobachtet werden, wie sich eine bestimmte Kategorie, ein bestimmtes Phänomen, in verschiedenen Sprachen manifestiert. In der Tat werden jedoch auch die Unterschiede betrachtet. Den beim Sprachvergleich aufkommenden Ähnlichkeiten können vier Ursachen zugrunde liegen (nach Comrie 1989: 286):

1) Zufall

2) Die zu vergleichenden Sprachen sind genetisch verwandt.

3) Die Sprachen stehen geografisch nah beieinander und haben Eigenschaften der anderen übernommen.

4) Diese Eigenschaft kann ein sprachliches Universal sein, sowohl absolut, als auch tendenziell.

Wenn man also im Rahmen der vergleichenden bzw. kontrastiven Linguistik arbeiten möchte, kann man auf der einen Seite den Vergleich eines bestimmten Phänomens (z. B. die Flexion des Nomens, die Tempusmarkierungen im verbalen Paradigma usw.) in zwei oder mehreren Sprachen anstreben, was eine induktive Vorgehensweise voraussetzt; auf der anderen Seite aber kann sich der Linguist als Ziel setzen, eine Kategorie oder eine abstraktere sprachliche Erscheinung (wie Ergativität, die verbale Serienbildung, die Kategorisierung in einem bestimmten semantischen Feld usw.) aufzuspüren, wobei man von einem allgemein typisierten Phänomen der menschlichen Sprache ausgeht, um dessen Realisierungen in bestimmten Einzelsprachen nachzugehen.

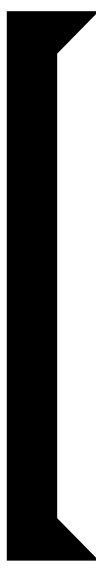

Die vergleichende linguisitik wirrde sich mit der typologischen KlassifizizerungyonSprachen beschïftigen, während die kontrassive linguisitik sich der Forschung überkonkreteAspekteinzweiodermehreeren Sprachen widmet.

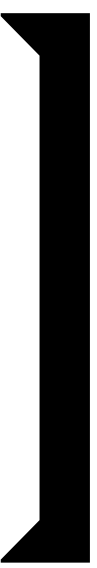

\section{Linguistische Universalien}

Einer der Kernbereiche der kontrastiven Linguistik besteht in der Suche nach sprachlichen Universalien: Merkmale, Eigenschaften, die jeder natürlichen Sprache gemeinsam sind. In dieser Hinsicht können Universalien als die Menge der Eigenschaften definiert werden, die von der menschlichen Sprache als Kommunikationssystem aufgewiesen wird. Einfache Beispiele dazu sind u.a. die Existenz von Vokalen und Konsonanten im phonetischen System, oder die Unterscheidung zwischen erster und zweiter Person Singular (ich/du) oder die Existenz der Satzmodi Aussage, Frage und Aufforderung.

Die Suche nach Universalien kann unter Einsatz zweier methodologischen Grundeinstellungen erfolgen: 
ind

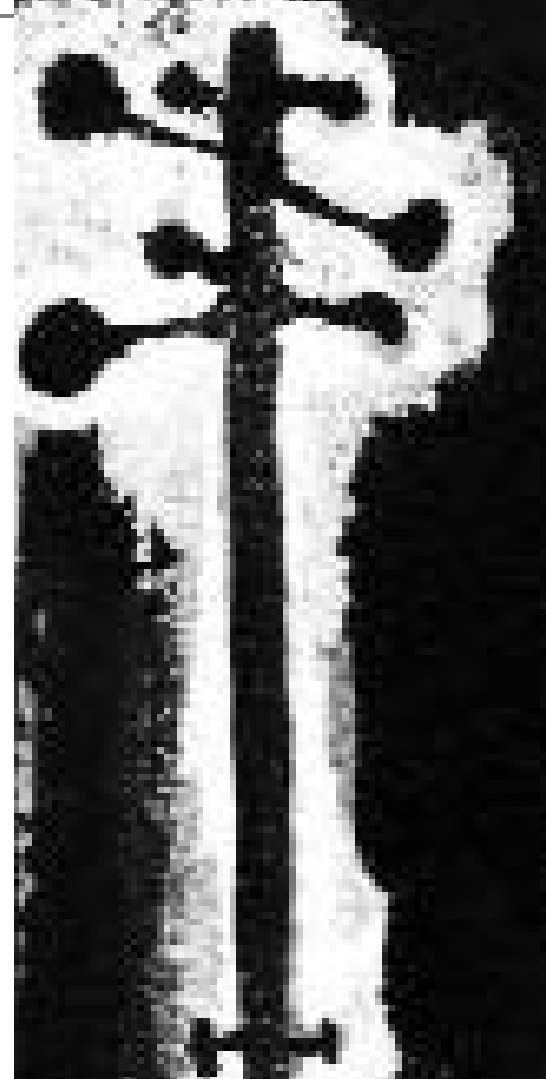

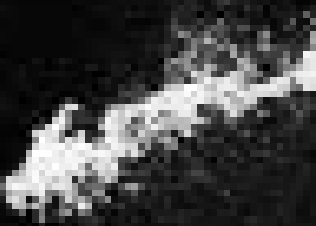

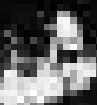

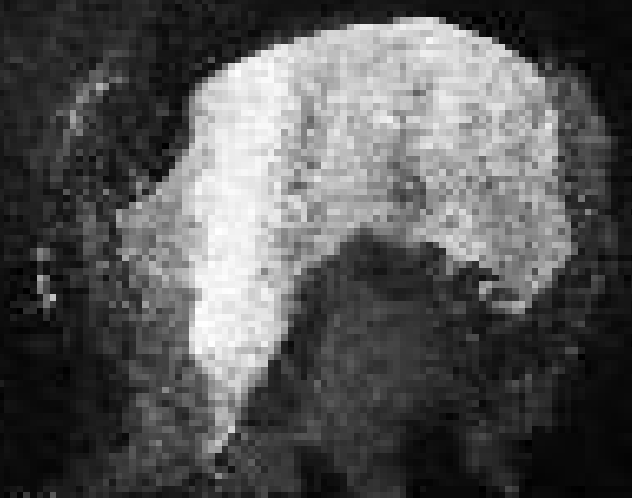


1) Greenberg vertritt die Ansicht, dass Universalien sich auf Datenerhebungen aus einem breiten Sprachenkatalog stützen und aus deren Analyse hervorgehen müssen.

2) Dagegen behauptet Chomsky, dass die Universalienforschung durch eingehende tiefe Analyse einer einzigen Einzelsprache durchgeführt werden kann. Es wird dann von abstrakten Strukturen ausgegangen, wie die syntaktische Tiefenstruktur der Generativen Transformationsgrammatik.

Im Folgenden behandeln wir, wie eine Klassifikation von Universalien unternommen werden kann.

Semiotische und grammatische, formale und substantive Universalien Auf dem Weg zur Klassifizierung von Universalien fällt die Unterscheidung zwischen semiotischen und grammatischen Universalien als erste aus. Semiotische Universalien umfassen Eigenschaften, die den Begriff menschlicher Sprache als Kommunikationssystem zusammen mit seinen physikalischen und physiologischen Beschaffenheiten ausmachen; die grammatischen beziehen sich auf jene Eigenschaften, die den konkreten Realisierungen (d. h. Eigenschaften oder Parameter) dieser menschlichen Sprache gemeinsam sind (Moreno Cabrera 1997: 53). Konsequent mit dieser Auffassung lassen sich grammatische Universalien nur in dem allgemeineren Rahmen der semiotischen einbinden.

Chomsky (1965) unterscheidet zwischen formalen und substantiven Universalien. Die formalen beziehen sich auf die abstrakten Eigenschaften von Sprachen, während die substantiven den physikalischen, physiologischen und psychischen Mitteln Rechnung tragen, deren sich die menschliche Sprache bedient.

Das folgende Schema verbildlicht die Kombinationen der beschriebenen Kategorien (Moreno Cabrera 1997: 54):

1. Semiotische Universalien

i. Formale: abstrakte Eigenschaften der menschlichen Sprache. Beispiel:

Jede menschliche Sprache ist ein referentielles System.

ii. Substantive: Eigenschaften der materiellen Koordinaten der menschlichen Sprache. Beispiel:

Jede menschliche Sprache bedient sich im Grunde des Lautes als Mittel zur Übertragung von Zeichen.

2. Grammatische Universalien:

i. Formale: Abstrakte Eigenschaften der

Grammatiken der natürlichen Sprachen. Beispiel: Grammatische Regeln sind von der Struktur abhängig.

ii. Substantive: Grammatische Eigenschaften von Sprachen. Beispiel:

Sprachen, die Personalflexion im Substantiv aufweisen, werden diese auch im Verb reflektieren.

Absolute und implikative Universalien

Universalien können auch auf Grund des Spektrums ihrer Geltung klassifiziert werden. Wenn es sich um Gesetze handelt, die in jeder menschlichen natürlichen Sprache gelten, wird man von absoluten Universalien sprechen. Zum Beispiel findet man in dem phonologischen System jeder Sprache Vokale und Konsonanten.
Wenn das Vorhandensein eines Merkmals in einer gegebenen Sprache die Existenz eines anderen bedingt, handelt es sich um implikative Universalien, die folgendermaßen definiert werden können: «Se dan entre dos (o más) características C1 y C2 de las lenguas humanas, de modo que si una lengua tiene C1 se predice que tendrá tambien C2» (Moreno Cabrera 1997: 67).

Implikative Universalien sind in jedem Bereich der Grammatik (Phonologie, Morphologie, Syntax, Semantik) vorhanden:

- Phonologische Universalien: Wenn es in einer Sprache nur drei Vokale gibt, werden es [a], [i] und [u] sein.

- Morphologische Universalien: Sollte eine Sprache unterschiedliche Affixe zulassen, wird die Häufigkeitsskala so sein: 1. Suffixe (am häufigsten), 2. Präfixe, 3. Infixe und 4. Zirkumfixe.

- Syntaktische Universalien: In Sprachen mit vorherrschender Verb-Subjekt-Objekt-Struktur (VSO) sind Objekte immer präpositionaler Natur (Greenberg 1966).

- Semantische Universalien: Wenn es in einer Sprache nur zwei Farbbezeichnungen gibt, wird die erste Unterscheidung schwarz-weiß (hell-dunkel) sein. Sollte eine dritte Farbe hinzukommen, wird diese rot sein. Bei vier oder fünf Farbbezeichnungen kommen gelb oder grün hinzu, an sechster Stelle kommt dann blau (Berlin/Kay 1969).

\section{Aktuelle Tendenzen der vergleichenden und kontrastiven Linguistik}

Die Suche nach Universalien in der Sprache dauert noch an und somit werden sprachtypologische Studien wie die oben erwähnten immer noch durchgeführt. Der technische Fortschritt und der internationale Handel haben die Welt «verkleinert» und so ist es heutzutage möglich, Sprachen zu erforschen, die zu einem früheren Zeitpunkt nicht aus dem Stadium des Wunschdenkens eines jeden Typologen herausgegangen wären. Lange hat die Klage der Typologen gewährt, man könne sich nur europäischer oder sonst sehr verbreiteter Sprachen annehmen, um diese $\mathrm{zu}$ erforschen und $\mathrm{zu}$ beschreiben. Heutzutage lassen sich Studien zum Yoruba (eine Kwa-Sprache aus Nigerien) genau so leicht finden wie Kantonesisch-Lehrwerke. Wie dem auch sei, die ausführliche Erforschung einer Sprache ist nach wie vor eine mühsame Arbeit. Andererseits sind durch die raschen Entwicklungen auf dem Gebiet der Informationstechnologien schriftliche wie akustische Belege in vielen unterschiedlichen Sprachen nur einen Mausklick entfernt.

Typologische und universalistische Studien haben nichts an Aktualität eingebüßt. Diesartige Studien reichen von dem einfachen Vergleich von phonetischen Beständen oder von der morphologischen Realisierung gewisser Funktionen (z. B. Ausdruck der Kasusformen) in einer Sprachfamilie, über Arbeiten syntaktischer (Satzbau und -typologie aufgrund der Stellung von Subjekt, Verb und Objekt, syntagmatische Beziehungen zwischen Satzgliedern) und semantischer (semantische Felder und ihre Beziehung im Rahmen der Prototypentheorie) Natur bis hin zu Studien zu interkultureller Pragmatik (vgl. Hernández Sacristán 1999).

Der Vergleich unter pragmatischen Kategorien impliziert naturbedingt, unterschiedliche Kulturen und deren Auffas- 
sung der Realität in Verbindung zu setzen. Bedenken wir, dass Fehler im sozialen Gebrauch einer Sprache zum Misslingen des kommunikativen Aktes führen können (vgl. Hernández Sacristán 1999: 26 f.).

Das Gespräch ist die prototypische Form des sprachlichen Gebrauchs, der zwischenmenschlichen Interaktion. Dank dem immer reger werdenden Kontakt zwischen den unterschiedlichen Sprachgemeinschaften hat das Interesse an vergleichenden Studien über Sprechakte oder Konversationsroutinen stark zugenommen. Die wachsende Reiselust, wie die Entwicklung in der Touristikbranche beweist, und die Verdichtung der wirtschaftlichen Beziehungen fordern die Intensivierung der persönlichen Kontakte in einem Maße, dass die Berührung zwischen Sprechern von typologisch, kulturell und geografisch sehr weit entfernten Sprachen etwas alltägliches geworden ist, wenn auch die Rolle des Englischen als lingua franca nicht verdrängt werden kann.

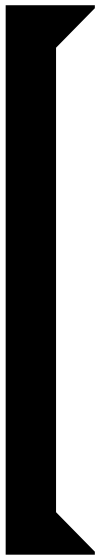

Sokannmanbeider kontrassivien Gessprächsanalyse fessistellen, dass Deutsche den Sprachturnus im kolloquidelen Gespräăh einhalten (es wird gewartet, bis der Gessprächspartner Signale zum Tuunwechsel gibt); dagegenen sind Unterbrechungen und Überlappungen im Spanischen sehr häuliti.

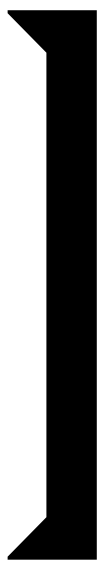

In den 90er Jahren ist Höflichkeit zu einem der Kernthemen der interkulturellen Pragmatik avanciert, denn es handelt sich dabei um eine pragmatische Kategorie, die in jedem Gespräch mit für jede Sprachgemeinschaft geltenden Regeln präsent ist. So kann man bei der kontrastiven Gesprächsanalyse zum Beispiel feststellen, dass Deutsche den Sprachturnus im kolloquialen Gespräch einhalten (es wird so lange gewartet, bis der Gesprächspartner eindeutige Signale zum Turnwechsel gegeben hat); dagegen sind Unterbrechungen und Überlappungen im Spanischen sehr häufig (vgl. Contreras Fernández, im Druck).

Ein weiteres Thema der Pragmatik, auf das kontrastiv eingegangen wird, ist der Vergleich von sprachlichen Elementen, welche die Haltung des Sprechers und die Beziehungen zwischen Äußerungen ausdrücken, u. a. Konnektoren, Modalpartikeln und Diskursmarkierungen. Da diese Wörter abstrakte Kategorien zum Ausdruck bringen, lassen sich oft keine zwischensprachlichen Äquivalenten finden, nicht mal zwischen typologisch nahestehenden Sprachen. Dies ist zum Beispiel der Fall bei den deutschen Modalpartikeln, die u. a. Einschätzungen des Sprechers über den propositionalen Sachverhalt vermitteln und für die sich keine Entsprechungen im Spanischen oder Englischen finden lassen (vgl. Ferrer Mora 2001a, 2001b, 2003b, 2004): Komm her! -Ven aquí- vs. Komm doch her! Ven ø aquí-); die Aufforderung im zweiten Satz erhält durch das doch eine zusätzliche Intensivierung und dadurch wird der Imperativsatz von der Modalpartikel nuanciert.

Die große Anziehungskraft der Pragmatik auf Forscher et alspiegelt sich in Studien über die Rolle der pragmatischen Inferenz wieder (vgl. Huan 1994, Bisang 2006). Die südostasiatischen Tonsprachen weisen das typologische Merkmal der Indetermination auf. Diese Eigenschaft besteht in dem Mangel an obligatorischen Kategorien, d. h., es gibt keine Flexion ${ }^{2}$ und sie ist mehreren Sprachen wie Chinesisch oder Thailändisch gemeinsam. Was in flektiven Sprachen wie Spanisch mittels grammatischer Beziehungen (auch durch die Syntax) ausgedrückt wird, bleibt im Chinesischen der pragmatischen Interpretation überlassen, denn man soll Argumente nicht erneut ausdrücken wenn diese bereits erwähnt wurden. Infolgedessen lässt sich das Subjekt eines Relativsatzes im Chinesischen (auch im Japanischen) nur aus dem Kontext ermitteln. Folgendes Beispiel aus Bisang $(2005)^{3}$ soll stellvertretend dieses Phänomen illustrieren:

a. [ch_de]rel rén comer ATR hombre «el hombre [que está comiendo]rel»

b. [ch_de]rel pínggu? comer ATR manzana «la manzana [que se come]rel»

c. [ch_de]rel fàngu?n comer ATR restaurante «el restaurante [en el cual se come]rel»

\section{Schlussbemerkungen und Desiderata}

Dieser Beitrag hat einen kurzen Überblick zur vergleichenden und kontrastiven Linguistik verschafft. Nach der Darstellung von Gegenstand und Methodik beider Subdisziplinen, wurde auf einige $z$. T. noch offene Fragen eingegangen, mit denen sich die vergleichende und kontrastive Linguistik beschäftigt. Darauf aufbauend ist der Begriff der linguistischen Universalien definiert und klassifiziert worden, eines der relevantesten Themen in diesem Bereich. Im letzten Abschnitt haben wir uns mit etlichen Aspekten aus aktuellen kontrastiven bzw. vergleichenden Forschungslinien beschäftigt, mit konkreten Beispielen aus der interkulturellen Pragmatik.

Typologische wie universalistische Studien werden heutzutage mit gezielten kontrastiven Studien in Verbindung gebracht. Die Tatsache, dass diese wissenschaftliche Ausrichtung in keinem spezifischen Studiengang an Universitäten als Hauptrichtung anerkannt wird, erklärt die Beschränkungen, denen diese Art Arbeit unterliegt.

Wie weit die Arbeit an der vergleichenden und kontrastiven Linguistik gehen kann, ist noch offen, denn es gibt bestimmt eine unbestimmte Zahl von noch zu entdeckenden Sprachen und die konkreten Aspekte, mit denen sich kontrastive Studien befassen, können methodologisch wie epistemologisch unterschiedlich durchgeführt werden. Jenseits der Linguistik können die Ergebnisse dieser Arbeit etliche Fragen anthropologischer, historischer und evolutionsgeschichtlicher Natur beleuchten. Die angeborene Neugierde des Menschen wirft die Frage 
immer wieder auf, nach den gemeinsamen Elementen in allen Sprachen und nach den Unterschieden, die hinter der sprachlichen Äußerung eines Sachverhalts stehen zu suchen. Die große Frage überhaupt wäre: Was macht die zwischenmenschliche Kommunikation erst möglich?
Dieser Beitrag kann unmöglich alle Aspekte und Entwicklungen in Disziplinen mit einer langen und sehr produktiven Tradition abdecken, das war auch nicht unser Ziel. Wir hoffen jedoch den Lesern dieses Artikels einen aussagekräftigen Überblick vermittelt zu haben

\section{Literatur}

Aitchinson, J. (1996), The Seeds of Speech. Language Origin and Evolution. Cambridge University Press, Cambridge.

Berlin, B., P. Kay, (1969), Basic Color Terms. Their Universality and Evolution. University of California Press, Berkeley, Los Angeles.

Bisang, W. (2001), 'Areality, grammaticalization and language typology. On the explanatory power of functional criteria and the status of Universal Grammar.' En Bisang, W. (ed.), Aspects of typology and universals. Akademie Verlag, Berlin: 175 -223.

Bisang, W. (2004), «Dialectology and typology - An integrative perspective.». En Kortmann, B., ed., Dialectology meets typology. Mouton de Gruyter, Berlin: $11-45$.

Bisang, W., (ed.)( 2001), Language typology and universals. Akademie Verlag, Berlin.

Bisang, W. (2006), «Southeast Asia as a Linguistic Area». Encyclopedia of Languages and Linguistics 2, 587-595.

Bisang, W. (2006), «Contact-Induced Convergence: Typology and Areality». Encyclopedia of Languages and Linguistics 2., 88101.

Bomhard, A., J.C. Kerns (1994), The Nostratic Macrofamily. A Study in Distant Linguistic Relationship. Mouton, Berlín.

Chomsky, N., (1965), Aspects of the Theory of Syntax. MIT, Cambridge.

Comrie, B. (1981), Language Universals and Linguistic Typology. Cambridge University Press, Cambridge.

Comrie, B., (1989), Universales del lenguaje y tipología lingüística. Gredos, Madrid. (Traducción de Augusta Ayuso).

Comrie, B. (ed.) (1987), The World's Major Languages. Croom Helm, London, Sydney.

Contreras Fernández, J., en prensa, El uso de la cortesía y las sobreposiciones en las conversaciones. Un análisis contrastivo alemánespañol. (Tesis doctoral). Universitat de València, Valencia.

Croft, W.A. (2003), Typology and Universals. Cambridge University Press, Cambridge.

Décsy, G. (1983), «A Preliminary List of the h-less Languages of the World: An Attempt at a Global Phoneme-Geography». En Décsy, G. (ed.), Global Linguistic Connections. Eurolingua, Bloomington: 7-33.

Ferrer Mora, H. (2001a) «Les partícules modals alemanyes i els seus equivalents en català des d'una perspectiva contrastiva». Caplletra 30: 95-110.

Ferrer Mora, H., (2001b), «Die deutschen Modalpartikeln und ihre Entsprechungen im Spanischen aus kontrastiver Sicht. Ein pragmatischer Fall». En: Heinrich, W./ Heiss, C., eds., Modalità e Substandard/Abtönung und Substandard. Università di Bologna, Forlì: 173-192.

Ferrer Mora, H. (2003a), «Espacio y movimiento: observaciones sobre la categorización espacial desde una perspectiva contrastiva». Estudios Filológicos Alemanes 3, Universidad de Sevilla: 231-243.

Ferrer Mora, H. (2003b), «La traducción de las partículas modales alemanas al español: ¿Una nueva clase lexemática en español?», en: Cerviño López, S./Delgado, T./Kaldemorgen, S.: Aprender a traducir. Walter Frey, Berlín: 55-68.

Ferrer Mora, H., 2004, «Las partículas modales alemanas y la traducción cero: el caso de denn". Estudios Filológicos Alemanes, 5. Sevilla: Kronos, 103-120.

Ferrer Mora, H., (2005), «Lingüística contrastiva y comparada", en Brady, I.K./Navarro Coy, M./Periñán Pascual, C. (eds.): Nuevas tendencias en lingüistica aplicada. Universidad Católica San Antonio, Murcia: 231249.

Fisiak, J. (1980), Contrastive Linguistics. Prospects and Problems. Mouton, Berlin.

Greenberg, J. H. (1966), Language Universals. With Special Reference to Feature Hierarchies. Mouton, La Haya, París.

Grimes, B. (ed.) (1992), Ethnologue. Languages of the World, Summer Institute of Linguistics. Dallas. (12 a edición)

Glück, H. (ed.) (2000), Metzler Lexikon Sprache. Metzler, Stuttgart, Weimer. ( $2^{\mathrm{a}}$ ed.)

Haspelmath, M. et al. (eds.) (2001), Langua- ge Typology and Language Universals. An International Handbook. Berlin/New York: de Gruyter. (= Handbücher zur Sprach-und Kommunikationswissenschaft;20,1;20,2).

Hernández Sacristán, C. (1999), Culturas y acción comunicativa. Introducción a la pragmática intercultural. Octaedro, Barcelona.

Huan, Y. (1994), The syntax and pragmatics of anaphora. Cambridge University Press, Cambridge.

Moreno Cabrera, J.C. (1990), Lenguas del mundo. Visor, Madrid.

Moreno Cabrera, J.C. (1997), Introducción a la lingüística. Enfoque tipológico y universalista. Síntesis, Madrid.

Pörings, R., U. Schmitz (1999), Sprache und Sprachwissenschaft. Narr, Tübingen.

Revert Sanz, V., B. Gallardo Paúls (2001), Glotonimia. Universitat de València, València.

\section{Anmerkungen}

1 Dieser Beitrag ist eine aktualisierte Version von Ferrer Mora (2005).

2 Verben sind morphologisch invariabel, Person, Numerus, Tempus und Aktionsart werden nicht markiert.

3 Das Beispiel entstammt einem Vortrag, den Prof. Bisang (Johannes-Gutenberg-Universität Mainz) im April 2005 an der Philologischen Fakultät der Universitat de València hielt. Aus der Diskussion ergab sich der wissenschaftliche Austausch, der zu den Arbeiten zur ersten Version dieses Beitrags wesentlich beigetragen hat. 
